\author{
United Nations Educational Scientific and Cultural Organization \\ and \\ International Atomic Energy Agency \\ THE ABDUS SALAM INTERNATIONAL CENTRE FOR THEORETICAL PHYSICS
}

\title{
HERMITIAN HARMONIC MAPS INTO CONVEX BALLS
}

\author{
Zhen Yang $\mathrm{Li}^{1}$ \\ Department of Mathematics, Zhejiang University, \\ Hangzhou 310027, Zhejiang, People's Republic of China \\ and \\ Xi Zhang ${ }^{2}$ \\ Department of Mathematics, Zhejiang University, \\ Hangzhou 310027, Zhejiang, People's Republic of China \\ and \\ The Abdus Salam International Centre for Theoretical Physics, Trieste, Italy.
}

\begin{abstract}
In this paper, we consider Hermitian harmonic maps from Hermitian manifolds into convex balls. We prove that there exist no non-trivial Hermitian harmonic maps from closed Hermitian manifolds into convex balls, and we use the heat flow method to solve the Dirichlet problem for Hermitian harmonic maps when the domain is compact Hermitian manifold with non-empty boundary. The case where the domain manifold is complete(noncompact) is also studied.
\end{abstract}

MIRAMARE - TRIESTE

July 2004

\footnotetext{
${ }^{1}$ lzymath@sina.com

2xizhang@zju.edu.cn
} 


\section{Introduction}

Let $(M, h)$ be a Hermitian manifold with Hermitian metric $\left(h_{\alpha \bar{\beta}}\right)$, and let $(N, g)$ be a Riemannian manifold with metric $\left(g_{i j}\right)$ and Christoffel symbols $\Gamma_{j k}^{i}$. A Hermitian harmonic map $u: M \rightarrow N$ satisfies the following elliptic system

$$
h^{\alpha \bar{\beta}} \frac{\partial^{2} u^{i}}{\partial z^{\alpha} \partial z^{\bar{\beta}}}+h^{\alpha \bar{\beta}} \Gamma_{j k}^{i} \frac{\partial u^{j}}{\partial z^{\alpha}} \frac{\partial u^{k}}{\partial z^{\bar{\beta}}}=0 .
$$

This system is more appropriate to Hermitian geometry than the harmonic map system since it is compatible with the holomorphic structure of the domain manifold in the sense that holomorphic maps are Hermitian harmonic maps when target manifolds are Kähler. Since (1.1) does not have a divergence structure nor a variational structure, it is analytically more difficult than harmonic system. It was firstly studied by Jost and Yau in [6], and was applied to study the rigidity of compact Hermitian manifolds. In [6], Jost and Yau consider the existence problems of Hermitian harmonic maps under the assumption that the target manifold $N$ is nonpositively curved. And Chen also studied the situation that the target manifold has nonempty boundary in [1]. In this paper, we consider the case where target manifolds are convex balls.

Let $N$ be a complete Riemannian manifold with sectional curvature bounded above by a positive constant $k$, and $B_{R}(O)$ be a geodesic ball of radius $R$ with center at fixed point $O \in N$. If $R<\frac{\pi}{2 \sqrt{k}}$, and $B_{R}(O)$ lies in the cut locus of $O$; then the geodesic ball $B_{R}(O)$ will be called by a convex ball.

Now let us fix some notation. Assume that $N$ is a Riemannian manifold. On $N$ we always choose the Levi-Civita connection which is compatible with the Riemannian structure. On $M$

now we choose the connection $\tilde{\nabla}$ such that it is compatible with the holomorphic structure on $M$ and torsion free. We denote the standard Beltrami-Laplacian by $\triangle$ and the Laplacian of the holomorphic torsion free connection by $\tilde{\triangle}$ respectively. Then one can define $\nabla d u(X, Y)$ by

$$
\nabla d u(X, Y)=\nabla_{Y} d u(X)-d u\left(\tilde{\nabla}_{X} Y\right)
$$

for any smooth map $u$ from $M$ to $N$. The torsion free assumption makes the above defined $\nabla d u(\cdot, \cdot)$ symmetric. And it is natural to define the tension fields of the map $u$ as

$$
\sigma(u)=\sigma(u)^{i} \frac{\partial}{\partial u^{i}}=h^{\alpha \bar{\beta}} \nabla d u\left(\frac{\partial}{\partial z^{\alpha}}, \frac{\partial}{\partial z^{\bar{\beta}}}\right)=h^{\alpha \bar{\beta}}\left(\frac{\partial^{2} u^{i}}{\partial z^{\alpha} \partial z^{\bar{\beta}}}+\Gamma_{j k}^{i} \frac{\partial u^{j}}{\partial z^{\alpha}} \frac{\partial u^{k}}{\partial z^{\bar{\beta}}}\right) \frac{\partial}{\partial u^{i}}
$$

If $u$ is a function on $M$, then

$$
\begin{gathered}
\widetilde{\triangle} u=h^{\alpha \bar{\beta}} \frac{\partial^{2} u}{\partial z^{\alpha} \partial z^{\bar{\beta}}} \\
\widetilde{\triangle} u-\frac{1}{2} \triangle u=<V, \nabla u>
\end{gathered}
$$


where $V$ is a well-defined vector field on $M$ and $\nabla$ is the Levi-Civita connection on $M$.

After giving some preliminaries in section 2, we will discuss the case where domain manifolds are compact. Firstly, we prove that there exist no non-trivial Hermitian harmonic map from closed Hermitian manifold into convex ball. In fact, we obtain the following theorem:

Theorem 1.1 Let $M$ be a closed (compact, without boundary)Hermitian manifold, and $N$ be a complete Riemannian manifold with sectional curvature bounded above by a positive constant $k$. Let $u: M \rightarrow N$ be a Hermitian harmonic map, and the image $u(M) \subset B_{R}(O)$. If $R<\frac{\pi}{2 \sqrt{k}}$, and $B_{R}(O)$ lies in the cut locus of $O$; then $u$ must be a constant map.

Secondly, we consider the case that domain manifold has non-empty smooth boundary. We use the heat flow method to prove the solubility of the Dirichlet problem for Hermitian harmonic maps. We obtain:

Theorem 1.2 Let $M$ be a compact Hermitian manifold with non-empty smooth boundary $\partial M$, and $N$ be a complete Riemannian manifold with sectional curvature bounded above by a positive constant $k$. Let $\phi: M \rightarrow N$ be a smooth map, and the image $\phi(M) \subset B_{R}(O)$. If $R<\frac{\arccos \frac{2 \sqrt{5}}{5}}{\sqrt{k}}$, and $B_{R}(O)$ lies in the cut locus of $O$; then there must exist a unique Hermitian harmonic map $u$, such that $\left.u\right|_{\partial M}=\left.\phi\right|_{\partial M}$.

Remark: We hope that the condition $R<\frac{\arccos \frac{2 \sqrt{5}}{5}}{\sqrt{k}}$ can be weakened by $R<\frac{\pi}{2 \sqrt{k}}$, but for technical reasons, in this paper we can only solve the Dirichlet problem for Hermitian harmonic map under this stronger condition.

As similar to harmonic maps, in section 4 we consider Hermitian harmonic maps from complete Hermitian manifolds into convex balls. The existence of Hermitian harmonic maps from complete Hermitian manifolds into Riemannian manifolds with non-positive curvature had been investigated by Lei Ni [8], Grunau and Kühnel [3]. In [3], Grunau and Kühnel introduced an invertibility condition on the holomorphic Laplace operator between suitable chosen function spaces. In fact, their proof shows that the solubility of the Poisson equation with respect to the holomorphic Laplace operator ensures the existence of Hermitian harmonic maps. Inspired by this, we will impose some conditions which are similar to those in [3], and we prove the existence of Hermitian harmonic maps from complete Hermitian manifolds satisfying these conditions into convex balls by using theorem 1.2 and the compact exhaustion method. 


\section{Preliminary Results}

We will solve (1.1) using the method of heat flow. Let $u: M \times \mathbf{R} \rightarrow \mathbf{N}$. We consider the following parabolic system:

$$
\begin{cases}h^{\alpha \bar{\beta}} \frac{\partial^{2} u^{i}}{\partial z^{\alpha} \partial z^{\beta}}+h^{\alpha \bar{\beta}} \Gamma_{j k}^{i} \frac{\partial u^{j}}{\partial z^{\alpha}} \frac{\partial u^{k}}{\partial z^{\beta}}=\frac{\partial u^{i}}{\partial t} & \text { for } z \in M \\ u(z, 0)=\phi(z) & \text { for } z \in \partial M, \quad 0 \leq t \leq \infty \\ u(z, t)=\phi(z) & \end{cases}
$$

where $\phi$ is a smooth map from $M$ to $N$ such that $\phi(M)$ is contained in the convex ball $B_{R}(O)$. By linearizing and using results about linear parabolic systems and the implicit function theorem, it follows in a standard manner that (2.1) has the short time existence.

In the following computation we need a Hessian comparison theorem by [4]: on the product $N \times N$ we introduce the Riemannian metric

$$
<X_{1} \oplus X_{2}, Y_{1} \oplus Y_{2}>:=<X_{1}, Y_{1}>+<X_{2}, Y_{2}>
$$

for $X_{i}, Y_{i} \in T_{y_{i}} N, y_{i} \in N, i=1,2$.

The distance function on $N$,

$$
\rho: N \times N \rightarrow \mathbf{R}
$$

is of class $C^{2}$ on $B_{R}(O) \times B_{R}(O)$ outside the diagonal. So we have:

Lemma 2.1([4][5]) $\forall y=\left(y_{1}, y_{2}\right) \in B_{R}(O) \times B_{R}(O), V \in T_{y}(N \times N)$,

$$
Q=\left(1-\cos \left(\sqrt{k} \rho\left(y_{1}, y_{2}\right)\right)\right) / k: B_{R}(O) \times B_{R}(O) \rightarrow \mathbf{R} .
$$

Then the Hessian of $Q$ admits the following estimates:

$$
\nabla^{2} Q(V, V) \geq \begin{cases}|V|^{2} & y_{1}=y_{2} \\ \frac{\leq \nabla Q(y), V>^{2}}{2 Q(y)}-k Q(y)|V|^{2} & y_{1} \neq y_{2}\end{cases}
$$

and

$$
\nabla^{2} Q(V, V) \geq(1-k Q(y))|U|^{2}
$$

if $V$ has the special form $U \oplus 0$ or $0 \oplus U$.

Multiplying the metric tensor by a suitable constant we may assume the upper bound of the sectional curvature of $N$ to be 1 throughout the rest part of this paper. We set $f(x, t)=$ $Q(u(x, t), O)$ and $e(u)=h^{\alpha \bar{\beta}} g_{i j} \frac{\partial u^{i}}{\partial z^{\alpha}} \frac{\partial u^{j}}{\partial z^{\beta}}$. At first, we will prove that the image of the solution of (2.1) contained in the convex ball $B_{R}(O)\left(R<\frac{\pi}{2}\right)$ under the flow such that we can use Lemma 2.1 for any time $t$. We have:

Lemma 2.2 Assume $u(z, t)$ is a solution of (2.1), then $\rho(u(z, t), O)<R$ for any $(z, t) \in$ $M \times \mathbf{R}$. 
Proof: Suppose not, so we can assume that at some point $\left(z_{0}, t_{0}\right) \in M \times \mathbf{R}, \rho\left(u\left(z_{0}, t_{0}\right), O\right)$ is equal to $R$ for the first time, so we have:

$$
\left.\frac{\partial}{\partial t} f\right|_{\left(z_{0}, t_{0}\right)} \geq 0,\left.\quad \nabla f\right|_{\left(z_{0}, t_{0}\right)}=0,\left.\quad \triangle f\right|_{\left(z_{0}, t_{0}\right)} \leq 0 .
$$

On the other hand, from (2.1) and Lemma 2.1, we compute at $\left(z_{0}, t_{0}\right)$

$$
\left(\tilde{\triangle}-\frac{\partial}{\partial t}\right) f=h^{\alpha \bar{\beta}} \nabla^{2} Q\left(\frac{\partial u}{\partial z^{\alpha}} \oplus 0, \frac{\partial u}{\partial z^{\bar{\beta}}} \oplus 0\right) \geq e(u) \cos R>0 .
$$

So we have the contradiction.

Next we will give estimates for $\left|u_{t}\right|^{2}:=\left|\frac{\partial u}{\partial t}\right|^{2}=g_{i j} \frac{\partial u^{i}}{\partial t} \frac{\partial u^{j}}{\partial t}$ and $e(u)$. By the assumption of the curvature, using the formulas (4), (7) in [6], we have

$$
\begin{gathered}
\left(\tilde{\triangle}-\frac{\partial}{\partial t}\right)\left|u_{t}\right|^{2} \geq\left|\nabla u_{t}\right|^{2}-2\left|u_{t}\right|^{2} e(u) \\
\left(\tilde{\triangle}-\frac{\partial}{\partial t}\right) e(u) \geq \frac{1}{2}\left|\nabla^{2} u\right|^{2}-2 e(u)(e(u)+c)
\end{gathered}
$$

where $c$ is a positive constant depending on the upper bound of both first and second derivatives of domain metric.

Let $b=1-\cos R_{0}, 0<R<R_{0}<\frac{\pi}{2}$. Hence $(b-f)$ is a positive bounded function. By (2.3) we can obtain

$$
\begin{array}{r}
\left(\tilde{\triangle}-\frac{\partial}{\partial t}\right) \frac{\left|u_{t}\right|^{2}}{(b-f)^{2}}=\frac{1}{(b-f)^{4}}\left[(b-f)^{2}\left(\tilde{\triangle}-\frac{\partial}{\partial t}\right)\left|u_{t}\right|^{2}+2\left|u_{t}\right|^{2}(b-f)\right. \\
\left.\cdot\left(\tilde{\triangle}-\frac{\partial}{\partial t}\right) f+2(b-f)<\nabla\left|u_{t}\right|^{2}, \nabla f>+3\left|u_{t}\right|^{2}|\nabla f|^{2}\right] \\
\geq \frac{1}{(b-f)^{4}}\left[\left(\left|\nabla u_{t}\right|^{2}-2\left|u_{t}\right|^{2} e(u)\right)(b-f)^{2}+2(1-f) e(u)\left|u_{t}\right|^{2}(b-f)\right. \\
\left.+2(b-f)<\nabla\left|u_{t}\right|^{2}, \nabla f>+3\left|u_{t}\right|^{2}|\nabla f|^{2}\right]
\end{array}
$$

So we get

$$
\begin{array}{r}
\left(\tilde{\triangle}-\frac{\partial}{\partial t}\right) \frac{\left|u_{t}\right|^{2}}{(b-f)^{2}} \geq \frac{1}{(b-f)^{4}}\left[\left|\nabla u_{t}\right|^{2}(b-f)^{2}+2(1-b)(b-f) e(u)\left|u_{t}\right|^{2}\right. \\
\left.+2(b-f)<\nabla\left|u_{t}\right|^{2}, \nabla f>+3\left|u_{t}\right|^{2}|\nabla f|^{2}\right]
\end{array}
$$

and

$$
\nabla\left|u_{t}\right|^{2}=(b-f)^{2} \nabla \frac{\left|u_{t}\right|^{2}}{(b-f)^{2}}-2 \frac{1}{b-f}\left|u_{t}\right|^{2} \nabla f
$$

By Schwarz inequality and (2.6) we have

$$
\left(\tilde{\triangle}-\frac{\partial}{\partial t}\right) \frac{\left|u_{t}\right|^{2}}{(b-f)^{2}} \geq \frac{1}{b-f}<\nabla \frac{\left|u_{t}\right|^{2}}{(b-f)^{2}}, \nabla f>
$$


from the maximum principle, $\left|u_{t}\right|$ is uniformly bounded.

Similarly we also have

$$
\tilde{\triangle}-\frac{\partial}{\partial t} \frac{e(u)+c}{(b-f)^{2}} \geq \frac{1}{b-f}<\nabla \frac{e(u)+c}{(b-f)^{2}}, \nabla f>
$$

where $c$ is the same constant in (2.4).

To get global $C^{1}$ estimate, from the formula (2.8), we know that it is sufficient to prove the boundary $C^{1}$ estimate. Firstly we need the following:

Lemma 2.3([6]) There exist $\delta_{0}>0$ and $R_{0}>0$ with the following property:

If $u$ is a solution of (2.1) for $0 \leq t \leq T$ and if for some $t_{0}, 0<t_{0} \leq T, u\left(B\left(x_{0}, R\right), t_{0}\right) \subset$ $B_{\delta}(p), x_{0} \in M, B\left(x_{0}, R\right) \subset M, 0<\delta \leq \delta_{0}$, for some $R, 0<R \leq R_{0}, p \in N,\left(B(q, r):=\left\{q^{\prime} \in M\right.\right.$ : $\left.d\left(q, q^{\prime}\right)\right)$, then

$$
\left|\nabla u\left(x_{0}, t_{0}\right)\right| \leq \frac{c \delta}{R} \quad(\nabla \text { denotes the spatial gradient })
$$

where $\delta_{0}, R_{0}$ and $c$ depend on the geometry of $M$ and $N$ and on $\sup _{B\left(x_{0}, R\right)}\left|u_{t}\left(x, t_{0}\right)\right|$.

Lemma 2.4 Let $u$ be a solution of (2.1) for $0 \leq t<T$, and the radius of convex ball $R<\frac{\pi}{4}$. Then there exits $0<t_{0}<T$ s.t.

$$
|\nabla u|(z, t) \leq c \quad(\nabla \text { denotes the spatial gradient })
$$

for $z \in \partial M, 0<t_{0} \leq t<T$, where $c$ is independent of $t$.

Proof : Lemma 2.3 has given interior gradient bound, and it consequently suffices to show if $d\left(z_{1}, z_{0}\right)=r, z_{1} \in \partial M, d\left(z_{2}, z_{0}\right) \leq r$, we have

$$
\rho\left(u\left(z_{1}, t\right), u\left(z_{2}, t\right)\right) \leq c_{1} r
$$

for some constant $c_{1}$ independent of $t$.

By Lemma 2.1 we know for any $z_{1}, z_{2} \in M, u\left(z_{1}, t\right), u\left(z_{2}, t\right)$ can be joined by a unique geodesic arc. We continue the geodesic arc from $u\left(z_{2}, t\right)$ to $u\left(z_{1}, t\right)$ beyond $u\left(z_{1}, t\right)$ until we reach a distance $\tau$ from $u\left(z_{1}, t\right)$ such that the corresponding point denoted by $q$ is contained in $B_{\frac{\pi}{4}}(O)$. We consider $L(z, t)=1-\cos \rho(u(z, t), q)$. Then by the choice of $q$, we have

$$
\left(\tilde{\triangle}-\frac{\partial}{\partial t}\right) L(z, t) \geq 0
$$

We then solve the following linear parabolic problem:

$$
\left\{\begin{array}{cl}
H: M \times \mathbf{R} \rightarrow \mathbf{R} & \\
\left(\tilde{\triangle}-\frac{\partial}{\partial t}\right) H(z, t)=0 & \\
H(z, 0)=L(z, 0) \quad \text { for } \quad z \in M \\
H(z, t)=L(z, t) \quad \text { for } \quad z \in \partial M, \quad 0 \leq t \leq \infty
\end{array}\right.
$$


since $L$ has smooth boundary values, so does $H$.

The maximum principle implies

$$
L(z, t) \leq H(z, t) \quad \text { for } \quad t \geq 0, z \in M
$$

Now by (2.11) and (2.12)

$$
\begin{aligned}
\rho\left(u\left(z_{1}, t\right), u\left(z_{2}, t\right)\right) & =\rho\left(u\left(z_{2}, t\right), q\right)-\rho\left(u\left(z_{1}, t\right), q\right) \quad \text { by the choice of } \mathrm{q} \\
& \leq \frac{1}{\sin \tau}\left(L\left(z_{2}, t\right)-L\left(z_{1}, t\right)\right) \\
& \leq \frac{1}{\sin \tau}\left(H\left(z_{2}, t\right)-H\left(z_{1}, t\right)\right)
\end{aligned}
$$

since $z_{1} \in \partial M$. From the theory of linear parabolic equations, the solution of (2.11) has a boundary Lipschitz bound. So we get (2.9).

Using (2.8) together with Lemma 2.4, we know $e(u)$ is also uniformly bounded, so we obtain the long time existence of the solution of the equation (2.1).

Let $u_{1}(x, t)$ and $u_{2}(x, t)$ are two sequences of maps from $M$ into the convex ball $B_{R}(O)$. Let

$$
\begin{aligned}
\psi(z, t) & =Q\left(u_{1}(z, t), u_{2}(z, t)\right) \\
f_{i}(z, t) & =Q\left(u_{i}, O\right) \\
\varphi(z, t) & =\sum_{i=1}^{2} \omega\left(f_{i}(z, t)\right) .
\end{aligned}
$$

Using lemma 2.1, one can check that when $u_{1}(z, t) \neq u_{2}(z, t)$,

$$
\left(\widetilde{\triangle}-\frac{\partial}{\partial t}\right) \psi \geq \frac{|\nabla \psi|^{2}}{4 \psi}-\psi \sum_{i=1}^{2} e\left(u_{i}\right)+d Q\left(\left(\sigma\left(u_{1}\right)-\frac{\partial u_{1}}{\partial t}\right) \oplus\left(\sigma\left(u_{2}\right)-\frac{\partial u_{2}}{\partial t}\right)\right)
$$

when $u_{1}(z, t)=u_{2}(z, t)$,

$$
\left(\widetilde{\triangle}-\frac{\partial}{\partial t}\right) \psi \geq \sum_{i=1}^{2} e\left(u_{i}\right)+d Q\left(\left(\sigma\left(u_{1}\right)-\frac{\partial u_{1}}{\partial t}\right) \oplus\left(\sigma\left(u_{2}\right)-\frac{\partial u_{2}}{\partial t}\right)\right),
$$

and,

$$
\begin{aligned}
\left(\widetilde{\triangle}-\frac{\partial}{\partial t}\right) \varphi & \geq \sum_{i=1}^{2}\left(\frac{1}{2} \omega^{\prime \prime}\left(f_{i}\right)\left|\nabla f_{i}\right|^{2}+\omega^{\prime}\left(f_{i}\right)\left(1-f_{i}\right) e\left(u_{i}\right)\right. \\
& \left.+\omega^{\prime}\left(f_{i}\right) d Q\left(\left(\sigma\left(u_{i}\right)-\frac{\partial u_{i}}{\partial t}\right) \oplus 0\right)\right) .
\end{aligned}
$$

Assume that $u_{1}(z, t)$ and $u_{2}(z, t)$ both satisfy $(2.1)$, and set function $\omega(s)=-\ln (1-s)$ in (2.14). By formulas (2.15), (2.16), (2.17), and the fact $\omega^{\prime \prime}=\omega^{\prime 2}$, one can check that when 
$u_{1}(z, t) \neq u_{2}(z, t)$

$$
\begin{aligned}
e^{-\varphi}\left(\tilde{\triangle}-\frac{\partial}{\partial t}\right) e^{\varphi} \psi & =\left(\tilde{\triangle}-\frac{\partial}{\partial t}\right) \psi+\psi\left(\tilde{\triangle}-\frac{\partial}{\partial t}\right) \varphi+<\nabla \varphi, \nabla \psi>+\frac{1}{2} \psi|\nabla \varphi|^{2} \\
& \geq \frac{|\nabla \psi|^{2}}{4 \psi}-\psi \sum_{i=1}^{2} e\left(u_{i}\right)+\frac{1}{2} \psi \sum_{i=1}^{2} \omega^{\prime \prime}\left|\nabla f_{i}\right|^{2} \\
& +\psi \sum_{i=1}^{2} \omega^{\prime}\left(1-f_{i}\right) e\left(u_{i}\right)+<\nabla \varphi, \nabla \psi>+\frac{1}{2} \psi|\nabla \varphi|^{2} \\
& \geq \frac{|\nabla \psi|^{2}}{4 \psi}+\frac{1}{2} \psi \sum_{i=1}^{2} \omega^{\prime \prime}\left|\nabla \psi_{i}\right|^{2}+\frac{1}{2}<\nabla \varphi, \nabla \psi> \\
& +\frac{1}{2} e^{-\varphi}<\nabla \varphi, \nabla\left(e^{\varphi} \psi\right)> \\
& \geq \frac{1}{2} \psi \sum_{i=1}^{2} \omega^{\prime \prime}\left|\nabla \psi_{i}\right|^{2}-\frac{1}{4} \psi|\nabla \varphi|^{2}+\frac{1}{2} e^{-\varphi}<\nabla \varphi, \nabla\left(e^{\varphi} \psi\right)> \\
& \geq \frac{1}{2} e^{-\varphi}<\nabla \varphi, \nabla\left(e^{\varphi} \psi\right)>
\end{aligned}
$$

When $u_{1}(z, t)=u_{2}(z, t)$, we have $\psi=\nabla \psi=0$. So,

$$
e^{-\varphi}\left(\tilde{\triangle}-\frac{\partial}{\partial t}\right) e^{\varphi} \psi \geq \sum_{i=1}^{2} e\left(u_{i}\right) \geq 0 .
$$

From (2.18) and (2.19), the maximum principle implies the uniqueness of the solution of the equation (2.1). So,we already have:

Proposition 2.5 If $\phi(M) \subset B_{R}(O), R<\frac{\pi}{4}$ and $B_{R}(O)$ lies in the cut locus of $O$, then the evolution equation (2.1) has a unique solution which exits for $0 \leq t<\infty$.

\section{Hermitian harmonic maps from compact manifolds into con- vex balls}

Firstly, we consider Hermitian harmonic map from a closed manifold(compact without boundary) to convex ball $B_{R}(O)$.

Proof of Theorem 1.1: Let $u: M \rightarrow N$ is a Hermitian harmonic map, that is, $u$ satisfies (1.1), and $u(M) \subset B_{R}(O), R<\frac{\pi}{2}$. Putting $f(z)=Q(u(z), O)$, we have

$$
\tilde{\triangle} f(z) \geq e(u) \cos \rho(u(z), O) \geq 0
$$

Since $M$ is closed, the function $f(z)$ must be constantly, hence $\rho(u(z), O)$ is a constant. We conclude that $u(M) \subset S_{r}(O)$, where $S_{r}(O)$ denotes a geodesic sphere of radius $r$ with center at $O$. And there must be a point $z_{0} \in M$ such that $u\left(z_{0}\right) \in S_{r}(O)$. We join $u\left(z_{0}\right)$ with $O$ by a geodesic arc. On this geodesic arc, we can choose a point $O^{\prime}$ which different with $O$ such that we can find another geodesic ball $B_{r^{\prime}}\left(O^{\prime}\right)$ satisfying $B_{r}(O) \subset B_{r^{\prime}}\left(O^{\prime}\right) \subset B_{R}(O)$. Setting $F^{\prime}(z, t)=1-\cos \rho\left(u(z), O^{\prime}\right)$, we also have $\tilde{\triangle} F^{\prime}(z, t) \geq 0$. Using the maximum principle again, 
we have $\rho\left(u(z), O^{\prime}\right)=r-\rho\left(O, O^{\prime}\right)=r^{\prime \prime}$, for any $z \in M$, i.e. $u(M) \subset S_{r^{\prime \prime}}\left(O^{\prime}\right)$. But it is easy to see that there only one point in $S_{r}(O) \cap S_{r^{\prime \prime}}\left(O^{\prime}\right)$, so $u(M)=u\left(z_{0}\right)$.

Proof of Theorem 1.2: First of all, we will show that if $\phi(M) \subset B_{R}(O)$,

$$
\left(\tilde{\triangle}-\frac{\partial}{\partial t}\right) \frac{\left|u_{t}\right|^{2}}{(b-f)^{2}} \geq 0
$$

where $R=\arccos \frac{2 \sqrt{5}}{5}<\frac{\pi}{4}, R_{0}=\arccos \frac{\sqrt{5}}{5}$, and $b=1-\cos R_{0}$. In fact, from (2.5) we have

$$
\begin{array}{r}
\left(\tilde{\triangle}-\frac{\partial}{\partial t}\right) \frac{\left|u_{t}\right|^{2}}{(b-f)^{2}} \geq \frac{1}{(b-f)^{4}}\left\{\left[\left|\nabla u_{t}\right|(b-f)-2\left|u_{t}\right||\nabla f|\right]^{2}\right. \\
\left.+2(1-b)(b-f)\left|u_{t}\right|^{2} e(u)-\left|u_{t}\right|^{2}|\nabla f|^{2}\right\} \\
\geq 2 \frac{1}{(b-f)^{4}}\left|u_{t}\right|^{2} e(u)\left[\cos R_{0}\left(\cos \rho-\cos R_{0}\right)-\sin ^{2} \rho\right]
\end{array}
$$

To get (3.2), it suffices to prove that $\cos R_{0}\left(\cos \rho(u, O)-\cos R_{0}\right)-\sin ^{2} \rho(u, O) \geq 0$, i.e.

$$
\cos \rho(u, O) \geq \sqrt{\frac{5}{4} \cos ^{2} R_{0}+1}-\frac{1}{2} \cos R_{0}
$$

And it is easy to see that when $R_{0}=\arccos \frac{\sqrt{5}}{5}$, the right side of (3.4) reaches its minimum $\frac{2 \sqrt{5}}{5}$. Therefore we have proved $(3.2)$, when $\rho(u(z), O) \leq R=\arccos \frac{2 \sqrt{5}}{5}$.

Now, we solve the following Dirichlet problem on $M([9]$, Ch5. proposition 1.8):

$$
\left\{\begin{array}{l}
\tilde{\triangle} v(z)=-\left.\frac{\left|u_{t}\right|^{2}}{(b-f)^{2}}\right|_{t=0} \\
\left.v(z)\right|_{\partial M}=0
\end{array}\right.
$$

Setting $w(z, t)=\int_{0}^{t} \frac{\left|u_{t}\right|^{2}}{(b-f)^{2}}(z, s) d s-v(z)$, By (3.2), we can see that

$$
\left\{\begin{array}{l}
\left(\tilde{\triangle}-\frac{\partial}{\partial t}\right) w(z, t) \geq 0 \\
\left.w(z, t)\right|_{\partial M}=0 \\
w(z, 0)=-v(z) \quad z \in M
\end{array}\right.
$$

Again by maximum principle we have

$$
\int_{0}^{t} \frac{\left|u_{t}\right|^{2}}{(b-f)^{2}} \leq \sup _{z \in M}|v(z)|<\infty
$$

Hence we conclude that there exists a sequence $\left\{t_{i}\right\}$ such that $\lim _{t_{i} \rightarrow \infty} u_{t}:=\lim _{t_{i} \rightarrow \infty} \frac{\partial u}{\partial t}=0$. Then, the standard elliptic regularity implies that there exists a subsequence $u\left(z, t_{i}\right)$ converge to a Hermitian harmonic map as $i$ goes to $\infty$.

Assume that $u_{1}(z)$ and $u_{2}(z)$ both are Hermitian harmonic maps satisfying the same boundary condition. Similar as (2.18) and (2.19), it is easy to obtain

$$
e^{-\varphi} \tilde{\triangle} e^{\varphi} \psi \geq \frac{1}{2} e^{-\varphi}<\nabla \varphi, \nabla\left(e^{\varphi} \psi\right)>
$$


or when $u_{1}(z)=u_{2}(z)$

$$
e^{-\varphi} \tilde{\triangle} e^{\varphi} \psi \geq 0
$$

where $\psi, \varphi$ is defined in (2.14). Then the maximum principle implies the uniqueness of Hermitian harmonic map.

\section{Hermitian harmonic maps from complete manifolds into con- vex balls}

In this section, we consider the existence of the Hermitian harmonic maps from some complete Hermitian manifolds into convex balls. Here the complete means complete, noncompact, and without boundary. We will use the solubility of Dirichlet problem which we have obtained in the above section and the compact exhaustion method to prove the existence of Hermitian harmonic map.

Next, we impose two invertibility assumptions on the holomorphic Laplace operator between suitably chosen function spaces.

Assumption 1: There exist positive numbers $\mu, \mu^{\prime}>0$ such that for every nonnegative function $\Theta \in C_{\mu}^{0}(M)$, there exists a nonnegative solution $v \in C_{\mu^{\prime}}^{0}(M)$ of

$$
\widetilde{\triangle} v=-\Theta
$$

Assumption 2: There exist positive numbers $p, p^{\prime}>0$ such that for every nonnegative function $f \in L^{p}(M) \cap C^{0}(M)$, there exists a nonnegative solution $u \in L^{p^{\prime}}(M) \cap C^{0}(M)$ of

$$
\widetilde{\triangle} v=-\Theta
$$

where $C_{\mu}^{0}(M)$ denote the space of continuous functions $v$ which satisfy that there exists $x_{0} \in M$ and a constant $C(v)$ such that $|v(x)| \leq C(v)\left(1+\operatorname{dist}\left(x, x_{0}\right)\right)^{-\mu}$.

Theorem 4.1. $\quad$ Let $M$ be a complete Hermitian manifold, $N$ be a complete Riemannian manifold with sectional curvature bounded above by a positive constant 1 , and $\phi: M \rightarrow N$ be a smooth map such that the image $\phi(M) \subset B_{R}(O)$. Here $R<\arccos \frac{2 \sqrt{5}}{5}$ and $B_{R}(O)$ lies in the cut locus of $O$. If the assumption 1 is satisfied with positive numbers $\mu>0, \mu^{\prime}>0$, and $\sigma(\phi) \in C_{\mu}^{0}(M)$, then then there exists a unique Hermitian harmonic map $u: M \rightarrow N$ such that $u$ is homotopic to $\phi$ and $(1-\cos (\rho(u, \phi))) \in C_{\mu^{\prime}}^{0}(M)$, where $\rho$ is the distant function on $N$.

If the assumption 2 is satisfied with positive numbers $p>0, p^{\prime}>0$, and $\sigma(\phi) \in L^{p}(M)$, then here exists a Hermitian harmonic map $u: M \rightarrow N$ such that $u$ is homotopic to $\phi$ and $(1-\cos (\rho(u, \phi))) \in L^{p^{\prime}}(M)$. 
Proof. Let $\left\{\Omega_{\alpha}\right\}$ be an exhausting sequence of compact subsets of $M$, i.e. they satisfy $\Omega_{\alpha} \subset \Omega_{\alpha+1}, \bigcup_{\alpha=1}^{\infty} \Omega_{\alpha}=M$. By Theorem 1.2 we have $\left\{u_{\alpha}\right\}$ which solve the Dirichlet problem

$$
\left\{\begin{array}{l}
\sigma\left(u_{\alpha}\right)=0 \\
\left.u_{\alpha}\right|_{\partial \Omega_{\alpha}}=\phi
\end{array}\right.
$$

By lemma 2.2, we know the maps $u_{\alpha}$ which we constructed must satisfy $u_{\alpha}\left(\Omega_{\alpha}\right) \subset B_{R}(O)$.

As above, we denote $f_{\alpha}=Q\left(u_{\alpha}, O\right), R_{0}=\arccos \frac{\sqrt{5}}{5}$, and $b=1-\cos R_{0}$. By similar computation which we have done in section 2 , and formula (2.4), it is not hard to see that

$$
\begin{aligned}
& \widetilde{\triangle} \frac{e\left(u_{\alpha}\right)}{\left(b-f_{\alpha}\right)^{2}} \geq \frac{1}{\left(b-f_{\alpha}\right)^{4}}\left\{2 e\left(u_{\alpha}\right)^{2}\left(b-f_{\alpha}\right)(1-b)-2 e\left(u_{\alpha}\right)^{2} \sin ^{2} \rho\left(u_{\alpha}, O\right)\right\}-2 c \frac{e\left(u_{\alpha}\right)}{\left(b-f_{\alpha}\right)^{2}} \\
& =\frac{2 e\left(u_{\alpha}\right)^{2}}{\left(b-f_{\alpha}\right)^{4}}\left(\cos ^{2} \rho\left(u_{\alpha}, O\right)+\cos \rho\left(u_{\alpha}, O\right) \cos R_{0}-\cos ^{2} R_{0}-1\right)-2 c \frac{e\left(u_{\alpha}\right)}{\left(b-f_{\alpha}\right)^{2}},
\end{aligned}
$$

where $c$ is the same constant in (2.4). Since $u_{\alpha}\left(\Omega_{\alpha}\right) \subset B_{R}(O)$, it is easily to check that

$$
\cos ^{2} \rho\left(u_{\alpha}, O\right)+\cos \rho\left(u_{\alpha}, O\right) \cos R_{0}-\cos ^{2} R_{0}-1 \geq 0
$$

So, we have

$$
\tilde{\triangle} \frac{e\left(u_{\alpha}\right)}{(b-f)^{2}} \geq-2 c \frac{e\left(u_{\alpha}\right)}{(b-f)^{2}}
$$

on $\Omega_{\alpha}$. Using Moser iteration(see [2] or [7]), one can easily check that the mean-value type inequality also holds here for $e\left(u_{\alpha}\right)$

$$
\sup _{x \in B\left(z_{0}, \frac{r}{2}\right)} e\left(u_{\alpha}\right) \leq c_{1}\left\|e\left(u_{\alpha}\right)\right\|_{L^{q}\left(B\left(z_{0}, r\right)\right.}
$$

for any $q>0$, and sufficiently large $\alpha$ such that $B\left(z_{0}, 2 r\right) \subset \Omega_{\alpha}$ and $c_{1}$ is a constant independent of $\alpha$. Therefore we can reduce the point-wise estimate of $e\left(u_{\alpha}\right)$ to an integral estimate of $e\left(u_{\alpha}\right)$.

In the following, we will discuss like that in [8] to estimate the integral of $e\left(u_{\alpha}\right)$ over geodesic ball $B\left(z_{0}, r\right)$. Let $G(x, y)$ be the positive Green function on $B\left(z_{0}, 2 r\right) \subset \Omega_{\alpha}$ satisfying the Dirichlet boundary condition. And $\theta$ is a cut-off function supported in $B\left(z_{0}, 2 r\right)$ with $\theta=1$ in $B\left(z_{0}, r\right)$. Then we have

$$
f_{\alpha}\left(x_{0}\right)=-\int_{B_{x_{0}}(2 r)} G(x, y) \triangle\left(\theta f_{\alpha}\right)
$$

By the Schwartz inequality we can write

$$
\begin{aligned}
\triangle\left(\varphi f_{\alpha}\right) & =\theta \triangle f_{\alpha}+2<\nabla f_{\alpha}, \nabla \theta>+f_{\alpha} \triangle \theta \\
& =\theta \tilde{\triangle} f_{\alpha}-\varphi<V, \nabla f_{\alpha}>+2<\nabla f_{\alpha}, \nabla \theta>+f_{\alpha} \triangle \theta \\
& =\theta e\left(u_{\alpha}\right) \cos \rho-\theta\|V\| \sqrt{2 e\left(u_{\alpha}\right)} \sin \rho-2|\nabla \theta| \sqrt{2 e\left(u_{\alpha}\right)} \sin \rho+f_{\alpha} \triangle \theta \\
& \geq \frac{1}{2} \theta e\left(u_{\alpha}\right) \cos \rho-2 \frac{\sin ^{2} \rho}{\cos \rho}\|V\|^{2} \theta-8 \frac{\sin ^{2} \rho}{\cos \rho} \frac{|\nabla \theta|^{2}}{\theta}+f_{\alpha} \triangle \theta
\end{aligned}
$$


So we have

$$
\begin{aligned}
f_{\alpha}\left(x_{0}\right) \leq & -\frac{1}{2} \int_{B_{x_{0}}(2 r)} G(x, y) \theta e\left(u_{\alpha}\right) \cos \rho+\int_{B_{x_{0}}(2 r)} G(x, y)\left(2 \frac{\sin ^{2} \rho}{\cos \rho}\|V\|^{2} \theta\right. \\
& \left.+8 \frac{\sin ^{2} \rho}{\cos \rho} \frac{|\nabla \theta|^{2}}{\theta}-f_{\alpha} \triangle \theta\right)
\end{aligned}
$$

Using the fact $f_{\alpha}$ is uniformly bounded, we can get

$$
\int_{B_{x_{0}}(r)} e\left(u_{\alpha}\right) \leq C
$$

Here the constant $C$ is independent of $\alpha$. Hence from (4.4) we know $e\left(u_{\alpha}\right) \leq C_{*}$ for some constant $C_{*}$ independent of $\alpha$. Then the standard elliptic theory shows that, by passing a subsequence, $u_{\alpha}$ converge uniformly over any compact sub-domain of $M$ to a smooth map $u$ such that $u$ is a solution of (1.1) on $M$.

Putting $u_{1}=u_{\alpha}, u_{2}=\phi$ in (2.14), i.e. $\psi(z)=Q\left(u_{\alpha}(z), \phi(z)\right)$, and taking $\omega(s)=-\frac{1}{2} \ln (1-$ $2 s)$, discussing like that in (2.18) (2.19), we have: when $\phi(z) \neq u_{\alpha}(z)$,

$$
\begin{aligned}
e^{-\varphi} \tilde{\triangle} e^{\varphi} \psi \geq & \psi \omega^{\prime}\left(f_{2}\right) d Q(\sigma(\phi) \oplus 0)+\frac{|\nabla \psi|^{2}}{4 \psi}-\psi \sum_{i=1}^{2} e\left(u_{i}\right)+\frac{1}{2} \psi \sum_{i=1}^{2} \omega^{\prime \prime}\left|\nabla \psi_{i}\right|^{2} \\
& +\psi \sum_{i=1}^{2} \omega^{\prime}\left(f_{i}\right)\left(1-f_{i}\right) e\left(u_{i}\right)+<\nabla \varphi, \nabla \psi>+\frac{1}{2} \psi|\nabla \varphi|^{2}+d Q(0 \oplus \sigma(\phi)) \\
\geq & -c_{2}\|\sigma(\phi)\|+\psi \sum_{i=1}^{2}\left[\omega\left(f_{i}\right)^{\prime}\left(1-f_{i}\right)-1\right] e\left(u_{i}\right) \\
& +\frac{1}{2} \psi \sum_{i=1}^{2} \omega^{\prime \prime}\left(f_{i}\right)\left|\nabla f_{i}\right|^{2}-\frac{1}{2} \psi|\nabla \varphi|^{2}
\end{aligned}
$$

where $c_{2}$ is a positive constant. Using the fact that $\omega^{\prime \prime}=2 \omega^{\prime 2}$, we have

$$
\begin{aligned}
e^{-\varphi} \tilde{\triangle} e^{\varphi} \psi & \geq-c_{2}(\|\sigma(\phi)\|)+\psi \sum_{i=1}^{2} \frac{f_{i}}{1-2 f_{i}} e\left(u_{i}\right) \\
& \geq-c_{2}(\|\sigma(\phi)\|)
\end{aligned}
$$

since $1-2 f_{i} \in\left[\frac{4 \sqrt{5}}{5}-1,1\right]$ by our assumption. Similarly, when $\phi(z)=u_{\alpha}(z)$, we also have

$$
e^{-\varphi} \tilde{\triangle} e^{\varphi} \psi \geq-c_{2}(\|\sigma(\phi)\|)
$$

By the assumption, and the definition in (2.14), one can check that $0 \leq \varphi \leq-\frac{1}{2} \ln \left(\frac{4 \sqrt{5}}{5}-1\right)$. When the assumption 1 is satisfied, we can find a non-negative function $v_{1} \in C_{\mu^{\prime}}^{0}(M)$, such that $\widetilde{\triangle} v_{1}=-c_{3}\|\sigma(\phi)\|$ in $M$, where $c_{3}=c_{2}\left(\frac{4 \sqrt{5}}{5}-1\right)^{-\frac{1}{2}}$. By (4.2), the coincidence of $u_{\alpha}$ and $\phi$ on $\partial \Omega_{\alpha}$, and the maximum principle, we have

$$
1-\cos \rho\left(u_{\alpha}, \phi\right) \leq v_{1}
$$

on $\Omega_{\alpha}$. Then,

$$
1-\cos \rho(u, \phi) \leq v_{1}
$$


on $M$. So, the Hermitian harmonic map $u$ which we have constructed must satisfy (1 $\cos (\rho(u, \phi))) \in C_{\mu^{\prime}}^{0}(M)$.

The uniqueness of solution $u$ with the mentioned properties can be proved by the same way as that in [3]. Let $\tilde{u}$ be an arbitrary Hermitian-harmonic map such that $(1-\cos (\rho(u, \phi))) \in C_{\mu^{\prime}}^{0}(M)$. Using the inequality $1-\cos (A+B) \leq 4(1-\cos A+1-\cos B)$ for any $A, B \in\left[0, \frac{\pi}{4}\right]$, we have

$$
0 \leq 1-\cos (\rho(u, \tilde{u})) \leq 4\{(1-\cos (\rho(u, \phi)))+(1-\cos (\rho(\tilde{u}, \phi)))\} \in C_{\mu^{\prime}}^{0}(M)
$$

Hence for every $\epsilon>0$ outside a sufficiently large ball $B\left(z_{0}, r\right)$ around an arbitrary $z_{0} \in M$ we have

$$
1-\cos (\rho(u, \tilde{u})) \leq \epsilon
$$

On the other hand, from formula (4.7) (4.8), we have

$$
\tilde{\triangle} e^{\varphi}(1-\cos (\rho(u, \tilde{u}))) \geq 0
$$

where we put $u_{1}=u, u_{2}=\tilde{u}$ in (2.14). By the maximum principle, we have $1-\cos (\rho(u, \tilde{u})) \leq$ $\left(\frac{4 \sqrt{5}}{5}-1\right)^{-1} \epsilon$ on all of $M$ for every $\epsilon>0$ and hence $1-\cos (\rho(u, \tilde{u}))=0$. This implies $u=\tilde{u}$.

When the assumption 2 is satisfied, we can find a non-negative function $v_{2} \in L^{p^{\prime}}(M)$, such that $\widetilde{\triangle} v_{1}=-c_{3}\|\sigma(\phi)\|$ in $M$. Discussing like that in the above, we have

$$
o \leq 1-\cos \rho(u, \phi) \leq v_{2}
$$

So, the Hermitian-harmonic map $u$ which we have constructed must satisfy $(1-\cos (\rho(u, \phi))) \in$ $L^{p^{\prime}}(M)$. The property that $u$ is homotopic to $\phi$ is obviously.

Remark: The assumption 1 was introduced by Grunau and Kühnel in [3], and they had constructed some examples which satisfy the assumption 1. Next, with help of the following two definitions, we want to discuss the assumption 2 on the holomorphic Laplace operator. We also discuss the uniqueness of Hermitian-harmonic maps from complete Hermitian manifolds into convex balls under some stronger conditions.

Definition 4.2. (Positive spectrum) Let $M$ be a complete Hermitian manifold, we say the holomorphic Laplace operator $\widetilde{\triangle}$ has positive first eigenvalue if there exists a positive number $c$ such that for any compactly supported smooth function $v$ one has

$$
\int_{M}(-\widetilde{\triangle} v) v \geq c \int_{M} v^{2}
$$

The supremum of these numbers $c$ will be denoted by $\widetilde{\lambda_{1}}(M)$. 
Definition 4.3. ( $L^{2}$-Sobolev inequality) Let $M$ be a m-dimensional(complex) complete Hermitian manifold $(m>1)$, we say the holomorphic Laplace operator $\widetilde{\triangle}$ satisfies $L^{2}$-Sobolev inequality if there exists a constant $S(M)$ such that for any compact supported smooth function $v$ one has

$$
\int_{M}(-\widetilde{\triangle} v) v \geq S(M)\left(\int_{M} v^{\frac{4 m}{2 m-2}}\right)^{\frac{2 m-2}{2 m}}
$$

Lemma 4.4. Let $M$ be a complete Hermitian manifold, and the holomorphic Laplace operator $\widetilde{\triangle}$ has positive first eigenvalue $\widetilde{\lambda_{1}}(M)$. Then for a nonnegative continuous function $\theta$ the equation

$$
\widetilde{\triangle} v=-\theta
$$

has a nonnegative solution $v \in W_{\text {loc }}^{2,2 m} \cap C_{\text {loc }}^{1, \beta}(M) \cap L^{p}(M)(0<\beta<1)$ if $\Theta \in L^{p}(M)$ for some $p \geq 2$.

Proof. We first solve the following Dirichlet problem on $\Omega_{\alpha}([9]$; Ch5, proposition 1.8)

$$
\left\{\begin{array}{l}
\widetilde{\triangle} v_{\alpha}=-\Theta, \\
\left.v_{\alpha}\right|_{\partial \Omega_{\alpha}}=0 .
\end{array}\right.
$$

Here $\Omega_{\alpha}$ is a exhaustion of $M$. First, by the maximum principle, we know that $v_{\alpha} \geq 0$. Now multiplying $v_{\alpha}^{p-1}$ on both-sides of the equation and integrating by parts we have that

$$
\begin{aligned}
\int_{\Omega_{\alpha}} \Theta v_{\alpha}^{p-1} & =\int_{\Omega_{\alpha}}\left(-\widetilde{\triangle} v_{\alpha}\right) \cdot v_{\alpha}^{p-1} \\
& =(p-1) \int_{\Omega_{\alpha}} v_{\alpha}^{p-2}\left|\nabla v_{\alpha}\right|^{2}-\int_{\Omega_{\alpha}} v_{\alpha}^{p-1}\left\langle V, \nabla v_{\alpha}\right\rangle
\end{aligned}
$$

On the other hand, using the assumption that $\widetilde{\lambda_{1}}(M)>0$, we have

$$
\begin{aligned}
\widetilde{\lambda_{1}}(M) \int_{\Omega_{\alpha}} v_{\alpha}^{p} & \leq \int_{\Omega_{\alpha}}\left(-\widetilde{\triangle} v_{\alpha}^{\frac{p}{2}}\right) v_{\alpha}^{\frac{p}{2}} \\
& =\left(\frac{p}{2}\right)^{2} \int_{\Omega_{\alpha}} v_{\alpha}^{p-2}\left|\nabla v_{\alpha}\right|^{2}-\frac{p}{2} \int_{\Omega_{\alpha}} v_{\alpha}^{p-1}\left\langle V, \nabla v_{\alpha}\right\rangle
\end{aligned}
$$

Adding (4.17) and (4.18), we have

$$
\frac{p}{2} \int_{\Omega_{\alpha}} \Theta v_{\alpha}^{p-1} \geq \frac{p}{2}\left(\frac{p}{2}-1\right) \int_{\Omega_{\alpha}} v_{\alpha}^{p-2}\left|\nabla v_{\alpha}\right|^{2}+\widetilde{\lambda_{1}}(M) \int_{\Omega_{\alpha}} v_{\alpha}^{p}
$$

From the above inequality, using Hölder inequality, we have

$$
\left(\int_{\Omega_{\alpha}} v_{\alpha}^{p}\right)^{\frac{1}{p}} \leq \frac{p}{2 \widetilde{\lambda_{1}}(M)}\left(\int_{M} \Theta^{p}\right)^{\frac{1}{p}}
$$

Using the interior $L^{p}$ estimates for the linear elliptic equation ([2], Theorem 9.11) we know that, over a compact sub-domain $\Omega$, there will be a uniform bound for $\left\|v_{\alpha}\right\|_{W^{2, p}(\Omega)}$. Therefore, using Rellich's compactness theorem, by passing to a subsequence we know that $v_{\alpha}$ will converge to 
a solution $v \in W_{l o c}^{2, p}(M) \cap L^{p}(M)$ on the manifold $M$. Since $\Theta \in L_{l o c}^{q}(M)$ for any $q \geq 2 m$, then the regularity theory for linear elliptic PDE implies that $v \in W_{l o c}^{2,2 m}(M) \cap C_{l o c}^{1, \beta}(M)$.

Replacing the Poincaré inequality (4.14) by the Sobolev inequality (4.15), and the test functions $v_{\alpha}^{p-1}$ by $v_{\alpha}^{a-1}$ (where $a=\frac{(m-1) p}{m-p}$ ) in the proof of above lemma, we can prove the following lemma.

Lemma 4.5. Let $M$ be a m-dimensional (complex) complete Hermitian manifold, and the holomorphic Laplace operator $\widetilde{\triangle}$ satisfy the $L^{2}$-Sobolev inequality (4.15). Then for a nonnegative continuous function $\Theta$ the equation

$$
\widetilde{\triangle} v=-\Theta
$$

has a nonnegative solution $u \in W_{l o c}^{2,2 m} \cap C_{l o c}^{1, \beta}(M) \cap L^{q}(M)(0<\beta<1)$ if $f \in L^{p}(M)$ for some $m>p \geq 2$, where $q=\frac{m p}{m-p}$.

The above two lemmas show that when the holomorphic Laplace operator $\widetilde{\triangle}$ has positive first eigenvalue (or satisfies the $L^{2}$ Sobolev inequality) then the condition 2 must be satisfied for some positive numbers $p, q$.

Corollary 4.6 Let $M$ be a complete Hermitian manifold, $N$ be a complete Riemannian manifold with sectional curvature bounded above by a positive constant 1 , and $\phi: M \rightarrow N$ be a smooth map such that the image $\phi(M) \subset B_{R}(O)$. Here $R<\arccos \frac{2 \sqrt{5}}{5}$ and $B_{R}(O)$ lies in the cut locus of $O$. If the holomorphic Laplace operator $\widetilde{\triangle}$ has positive first eigenvalue $\widetilde{\lambda_{1}}(M)$, and $\sigma(\phi) \in L^{p}(M)$ for some $p \geq 2$. Then here exists a Hermitian harmonic map $u: M \rightarrow N$ such that $u$ is homotopic to $\phi$ and $(1-\cos (\rho(u, \phi))) \in L^{p}(M)$. In particular, if the difference vector fields $V$ in (1.4) satisfies $\|V\|_{L^{\infty}}<\infty$, then, in this class, the solution is unique.

Proof. The existence of Hermitian harmonic maps is a corollary of theorem 4.1 and lemma 4.4. Let $\tilde{u}$ an arbitrary Hermitian harmonic map satisfying the properties which mentioned in the assumption. By the formula (4.10), we have

$$
1-\cos \rho(u, \tilde{u}) \in L^{p}(M) .
$$

Using the formula (4.12), and discussing like that in [8] (pp. 346-347), we have

$$
\int_{M}(1-\cos \rho(u, \tilde{u}))^{p} \leq 0
$$

which implies the uniqueness. 
Corollary 4.7 Let $M$ be a m-dimensional (complex) complete Hermitian manifold, $N$ be a complete Riemannian manifold with sectional curvature bounded above by a positive constant 1 , and $\phi: M \rightarrow N$ be a smooth map such that the image $\phi(M) \subset B_{R}(O)$. Here $R<\arccos \frac{2 \sqrt{5}}{5}$ and $B_{R}(O)$ lies in the cut locus of $O$. If the holomorphic Laplace operator $\widetilde{\triangle}$ satisfy the $L^{2}$-Sobolev inequality, and $\sigma(\phi) \in L^{p}(M)$ for some $p \in[2, m)$. Then there exists a Hermitian harmonic map $u: M \rightarrow N$ such that $u$ is homotopic to $\phi$ and $(1-\cos (\rho(u, \phi))) \in L^{q}(M)$, where $q=\frac{m p}{m-p}$. In particularly, if the difference vector fields $V$ in (1.4) satisfies $\|V\|_{L^{\infty}}<\infty$, then, in this class, the solution is unique.

Proof. We only need to prove the uniqueness of the solution. First, we will use the above $L^{2}$-Sobolev inequality to deduce a mean-value inequality of functions $v$ which satisfy

$$
\widetilde{\triangle} v \geq 0
$$

Let $0<\delta_{1}<\delta_{2} \leq 1$, and $\theta \in C_{0}^{\infty}\left(B\left(z_{0}, 2 r\right)\right)$ be the cut-off function

$$
\theta(x)= \begin{cases}1 ; & x \in B\left(z_{0},\left(1-\delta_{2}\right) r\right) \\ 0 ; & x \in B\left(z_{0}, 2 r\right) \backslash B\left(z_{0},\left(\left(1-\delta_{1}\right) r\right)\right.\end{cases}
$$

$0 \leq \theta(x) \leq 1$ and $|\nabla \theta| \leq 2\left(\delta_{2}-\delta_{1}\right)^{-1} r^{-1}$.

Let $A \geq 2$, multiply $v^{A-1} \theta$ on both sides of the formula (4.23) and integrate by parts. Then

$$
\begin{aligned}
& -\frac{A-1}{2} \int_{M}|\nabla v|^{2} v^{A-2} \theta^{2}-\int_{M}\langle\nabla v, \nabla \theta\rangle v^{A-1} \theta \\
& +\int_{M}\langle V, \nabla v\rangle v^{A-1} \theta^{2} \geq 0 .
\end{aligned}
$$

Using the Sobolev inequality that

$$
\int_{M}\left(-\widetilde{\triangle} v^{\frac{A}{2}} \theta\right) v^{\frac{A}{2}} \theta \geq S(M)\left(\int_{M}\left(v^{\frac{A}{2}} \theta\right)^{\frac{2 m}{m-1}}\right) \frac{m-1}{m},
$$

we have

$$
\|V\|_{L^{\infty}} \int_{M} v^{A} \theta|\nabla \theta|+\frac{1}{2} \int_{M} v^{A}|\nabla \theta|^{2} \geq S(M)\left(\int_{M} v^{A \frac{m}{m-1}} \theta^{\frac{2 m}{m-1}}\right)^{\frac{m-1}{m}},
$$

By the Schwarz inequality, then

$$
\int_{B\left(z_{0},\left(1-\delta_{2}\right) r\right)} v^{A\left(1+\frac{1}{m-1}\right)} \leq c_{4}\left\{\left(\delta_{2}-\delta_{1}\right)^{-2} r^{-2} \int_{B\left(z_{0},\left(1-\delta_{1}\right) r\right)} v^{A}\right\}^{1+\frac{1}{m-1}}
$$

Using the Moser's iteration, we have the following mean-value inequality.

$$
\|v\|_{L^{\infty}\left(B\left(z_{0}, \frac{1}{2} r\right)\right)} \leq C_{5}\left(r^{-2 m} \int_{B\left(z_{0}, r\right)} v^{A}\right)^{\frac{1}{A}} .
$$

Here $c_{5}$ is a constant depending only on $A, S(M)$, and $\|V\|_{L^{\infty}(M)}$. Let $\tilde{u}$ an arbitrary Hermitian harmonic map satisfying the properties which mentioned in the assumption. As in corollary 4.6, we have $1-\cos \rho(u, \tilde{u}) \in L^{q}(M)$. Then the formula (4.12) and the mean-value inequality imply that $1-\cos \rho(u, \tilde{u}) \equiv 0$. Hence $u \equiv \tilde{u}$. 


\section{$5 \quad$ References}

[1] Chen,J.Y., A boundary value problem for Hermitian harmonic maps and applications, Proc.Amer.Math.Soc. 124(1996), 2853-2862.

[2] D.Gilbarg,\& N.S.Trudinger, Elliptic partial differential equations of second order, 2nd edition, Springer-Verlag, 1983.

[3] Grunau,H.C. \& Kühnel,M., On the existence of Hermitian-harmonic maps from complete Hermitian to complete Riemaniann manifolds, Preprint.

[4] Jäger,W., \& Kaul,H., Uniqueness and stability of harmonic maps and their Jacobi fields. Manuscripta Math. 28 (1979), 269-291.

[5] Jost,J., Harmonic Mappings between Riemannian Manifolds. Proceedings of the Centre for Mathematic Analysis, 4. Australian National University,Canberra, 1984.

[6] Jost,J., \& Yau,S.T., A nonlinear elliptic system and rigidity theorem, Acta Math. 170 (1993), 221-254.

[7] Li,P., Lecture notes on geometric analysis, Research Institute of Mathematics, Global Analysis Center Seoul National University, 1993.

[8] Ni,L., Hermitian harmonic maps from complete Hermitian manifolds to complete Riemannian manifolds, Math.Z. 232 (1999), 331-355.

[9] M.E.Taylor, Partial differential equations I, Applied Mathematical Sciences 115, SpringerVerlag New York Berlin Heidelberg. 